\title{
FINE STRUCTURE OF THE FIBROUS SHEATH OF STALLION SPERM TAILS
}

\author{
STIG EINARSSON AND LENNART NIGANDER \\ Departments of Obstetrics and Gynaecology, and of Anatomy and Histology, \\ Royal Veterinary College, Stockholm, Sweden
}

(Received 9th fune 1967)

The fine structure of the sperm tail has been studied in many mammals. The tail is built of an axial filament complex and nine outer, coarse, longitudinal fibres (Bradfield, 1955). These fibres are surrounded by a mitochondrial sheath in the middle piece and by a fibrous sheath in the principal piece. This report will describe the character of the fibrous sheath and its development in the horse.

Material was obtained at slaughter or by castration from the testis and the cauda epididymidis of three normal stallions. After fixation in a $2 \%$ buffered solution of osmium tetroxide with sucrose (Millonig, 1961), it was embedded in Epon (Luft, 1961). Some blocks were treated with 1\% phosphotungstic acid in alcohol during this procedure. Thin sections were stained with uranyl acetate and studied in a Siemens Elmiskop I.

The development of the fibrous sheath takes place as described by Nicander (1962) in some other mammals. It starts during the cap phase of spermiogenesis. Two longitudinal strands of opaque material form inside the plasma membrane along fibres 3 and 8 of the tail primordium. Later these strands become interconnected by many semicircular 'ribs' of similar density. The strands eventually form denser, longitudinal pillars connected by coarse ribs. Cross-sections of the tail in epididymal spermatozoa (Pl. 1, Fig. 1) show the pillars as slight thickenings of the fibrous sheath opposite fibres 3 and 8 . The pillars are most distinct in the proximal part of the principal piece. The sheath often appears, even in rather thick sections, to be interrupted by empty spaces, suggesting defects in the ribs. This arrangement is also seen in immature testicular spermatozoa. Tangential sections of the sheath (Pl. 1, Fig. 2) invariably show the ribs to be unbroken, but broad and often branching and interconnecting. They run a rather oblique course relative to the plane of a cross-section of the tail and are separated by broader interspaces than in other mammalian species described so far. A similar tangential section of a pig sperm tail is shown for comparison (Pl. 1, Fig. 3).

The development of the fibrous sheath in horse spermatozoa shows no special features. In principle, its structure, in mature spermatozoa, also corresponds to that of other mammalian species, as established by Telkka, Fawcett \& Christensen (1961) and summarized by Fawcett (1965). Obviously, the impression of 'defects' in many cross-sections of horse sperm tails is caused by the very oblique course of many ribs and the unusually broad interspaces 
between them. The 'empty' part of the sheath in cross-section is just an area where the whole thickness of the section is occupied by an interspace between two ribs. In some other species, very thin cross-sections may actually show less opaque areas in the sheath, for similar reasons. In horse sperm tails, the phenomenon is so striking that it might give a false impression of an abnormality in the structure of the sperm tail.

\section{REFERENCES}

Bradfield, J. R. G. (1955) The fibre patterns in animal flagella and cilia. Symp. Soc. exp. Biol. 9, 306. FAWCETT, D. W. (1965) The anatomy of the mammalian spermatozoon with particular reference to the guinea pig. Z. Zellforsch. mikrosk. Anat. 67, 279.

LuFT, J. H. (1961) Improvements in epoxy resin embedding methods. F. biophys. biochem. Cytol. 9, 409. MilloniG, G. (1961) Advantages of a phosphate buffer for $\mathrm{OsO}_{4}$ solutions in fixations. 7. appl. Physiol. $32,1637$.

NiCANDER, L. (1962) Development of the fibrous sheath of the mammalian sperm tail. Vth int. Conf. for Electron Micros. M-4.

Telkxa, A., Fawcett, D. W. \& Christensen, A. K. (1961) Further observations on the structure of the mammalian sperm tail. Anat. Rec. 141, 231. 


\section{PLATE 1}
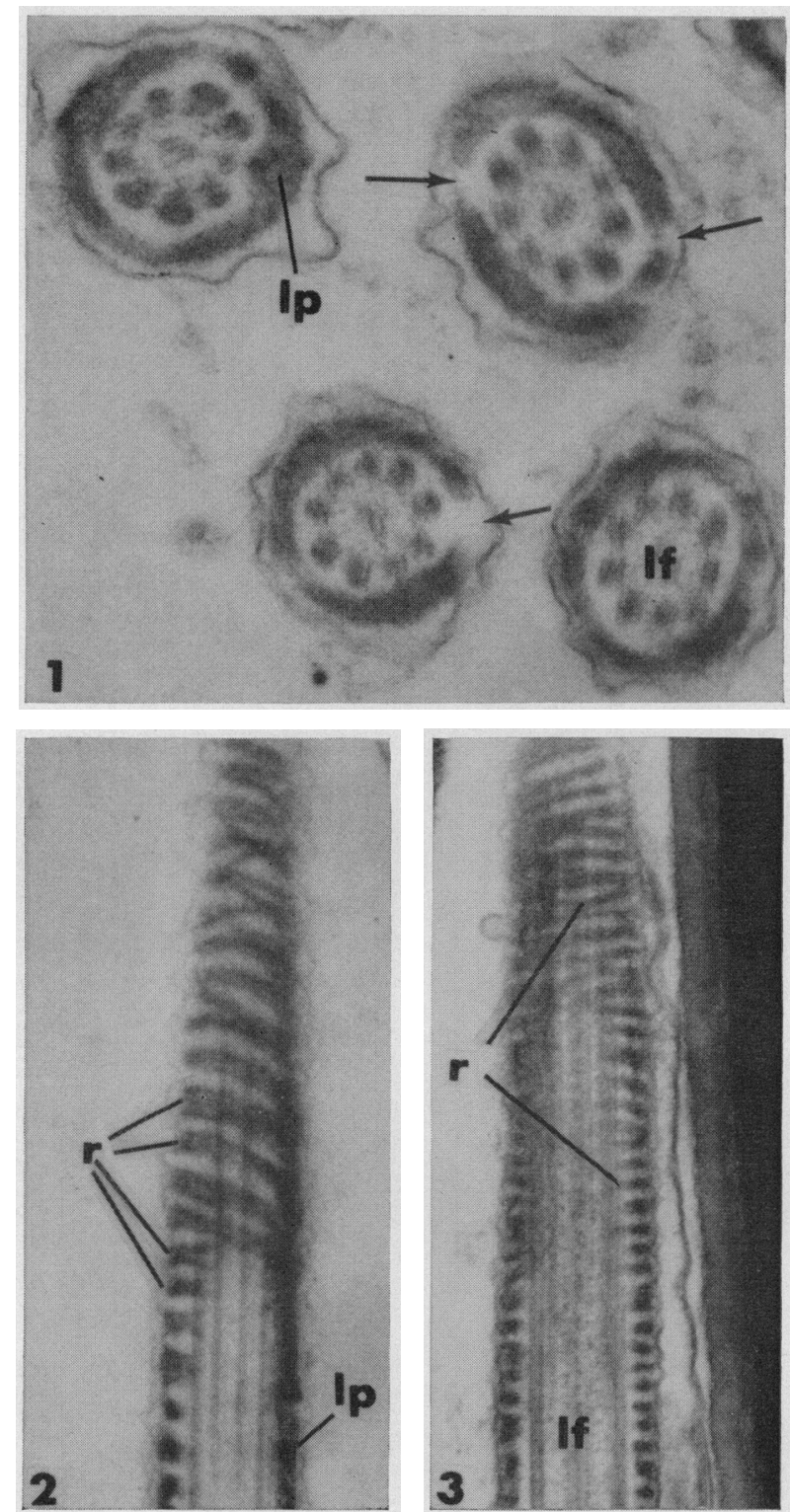

If $=$ longitudinal tail fibres, $1 \mathrm{p}=$ longitudinal pillar, $\mathrm{r}=$ ribs of the fibrous sheath.

Fig. 1. Cross-sections of principal pieces of four horse spermatozoa, showing 'defects' (arrows) of the fibrous sheath. Uranyl acetate. $\times 55,000$.

Fiç. 2. Obliquely longitudinal section of the principal piece of a horse sperm tail, showing the character of the ribs. Phosphotungstic acid. $\times 35,000$.

Fig. 3. Similar section of a pig sperm tail, for comparison. Lranyl acetate. $\times 40,000$. 